\title{
Biochemical response to hyperbaric oxygen treatment of a transhemispheric penetrating cerebral gunshot injury
}

\section{Eric Peter Thelin ${ }^{1}$, Bo-Michael Bellander ${ }^{1}{ }^{*}$ and Michael Nekludov ${ }^{2}$}

1 Section for Neurosurgery, Department of Clinical Neuroscience, Karolinska Institutet, Stockholm, Sweden

2 Department of Pharmacology and Physiology, Karolinska Institutet, Stockholm, Sweden

\author{
Edited by: \\ Edward Manno, Cleveland Clinic, USA \\ Reviewed by: \\ Laurie F. McWilliams, Cleveland \\ Clinic, USA \\ Ira Chang, Swedish Medical Center, \\ USA

\section{${ }^{*}$ Correspondence:} \\ Bo-Michael Bellander, Department of \\ Neurosurgery, R3:02, Karolinska \\ University Hospital Solna, S-17176 \\ Stockholm, Sweden \\ e-mail: bo-michael.bellander@ \\ karolinska.se
}

Hyperbaric oxygen (HBO) therapy has been suggested a treatment option in order to reduce the development of secondary insults succeeding traumatic brain injury. This case report studied the course of a 23-year-old gentleman with a close range transhemispheric gunshot wound. The biochemical parameters, using a multi-modal monitoring in the neuro-intensive care unit, improved following HBO treatment.

Keywords: hyperbaric oxygen, multi-modal monitoring, penetrating brain injury, human, microdialysis

\section{INTRODUCTION}

A 23-year-old male, previously healthy, was admitted to the emergency room following a cerebral gunshot wound, at close range, from a hand gun. On admission, the patient presented with a Glasgow Coma Score (GCS) of 14 (E4 + M6 + V4), obeyed commands, had normal pupil responses and extremity movement, yet was agitated and restless. Respiratory and circulatory parameters were stable. The entrance was located just behind the right ear, no exit wound was visible. Subsequently, the patient was sedated, intubated, and a full body CT-scan was performed, revealing no extracranial injuries. A CT-scan of the head revealed several bone fragments in the right temporal lobe below the entrance of the bullet (Figure 1A), which progressed through the brain with a bihemispheric central and transventricular trajectory. Along the route, intraparenchymal- and subarachnoid-hemorrhages were present (Figure 1B). The patient underwent wound revision and monitoring neurosurgery, receiving a Licox ${ }^{\circledR}$ brain tissue oximetry device (PBtO2; Integra LifeSciences, Plainsboro, NJ, USA), an intracerebral microdialysis catheter (CMA70, Microdialysis $\mathrm{AB}$, Stockholm, Sweden), an intracranial pressure (ICP) device (Codman ${ }^{\circledR}$ DePuy Synthes, Johnson \& Johnson Medical, New Brunswick, NJ, USA), and an extra-ventricular drain (Medtronic, Minneapolis, MN, USA). The patient was transferred to the neurointensive care unit for further treatment. During the following day, the patient deteriorated with an increased ICP, increased intracerebral lactate:pyruvate ratio (LPR), and increased serum levels of the biomarker S100B (Figure 2). This prompted an increase in administration of sedatives (propofol and midazolam), iterative doses of hypertonic saline, and infusion of pentobarbiturates. Despite this, the ICP remained elevated. The neurosurgeon on call decided to perform a hemicraniectomy in order to improve the intracranial conditions. Following the procedure, the ICP returned to normal levels $(<20 \mathrm{mmHg}$ ), although the $\mathrm{LPR}$ remained high $(>40)$ (Figure 2 ). The post-operative CT-scan revealed hypodense areas, indicating edema or that the tissue was at risk for ischemia, primarily in the cerebral areas supplied by the posterior circulation (Figure 3).

On day 7 post-trauma, the patient deteriorated further, with increasing LPR and S100B levels, yet normal cerebral oxygenation $(\mathrm{PBtO} 2>15 \mathrm{~mm} \mathrm{Hg})$, hence not indicating ischemia, though perhaps an ongoing mitochondrial dysfunction with a metabolic crisis. This lead to the initiation of hyperbaric oxygen (HBO) treatment ( $75 \mathrm{~min}, 2.8$ bar with two air-brakes, followed by a stage-wise decompression during $40 \mathrm{~min}$ ), which had an imminent, and stabilizing, effect on the LPR (Figures 2 and 4 ). The ICP remained normal $(<20 \mathrm{mmHg}$ ) throughout the $\mathrm{HBO}$ treatment period. The $\mathrm{PBtO} 2$ was not monitored during the $\mathrm{HBO}$, because of technical issues, but showed a sustained increase to levels above $20 \mathrm{mmHg}$ after the completed hyperbaric treatment, allowing the decrease of fraction of inspired oxygen (FiO2) (Figure 5). The secondary increase of S100B stopped, and was followed by a steady decline (Figure 2). The focal LPR is measured using the microdialysis technique, which is compatible to use inside the hyperbaric chamber. However, the measured levels may not be completely reliable during the compression and decompression phases, since the speed of perfusion is influenced by the surrounding pressure changes. The patient received two further $\mathrm{HBO}$ treatments, same type and duration, on day 8 and day 10 post-trauma. In order to visualize the changes before and after $\mathrm{HBO}$ treatment, Figure 4 shows LPR, $\mathrm{PBtO} 2$, and ICP during the 3 days of $\mathrm{HBO}$ treatment. The LPR has the highest level after the HBO treatment, which is a single sample, and thus presumably false. Following this peak, there is a sustained period of lower LPR samples.

Day 16 after injury, the patient was provided with a tracheostomy. A MRI on day 16 showed bilateral temporal damage with cytotoxic edema along the trajectory, yet no ischemic injury or permanent damage to the frontal lobes (Figure 6). Neurophysiological examination on day 27 revealed signs of bilateral cortical 
blindness (no visually evoked potentials detected), no signs of brain stem damage (normal brainstem auditory evoked potentials, BAEP), and no irregularities on the electromyogram (EMG). The patient himself was not aware of being blind.

On day 36, the patient was discharged from NICU to an intermediate neurosurgical ward.

The patient started physiotherapy and at day 38 after injury, he was able to move with support. At discharge from the

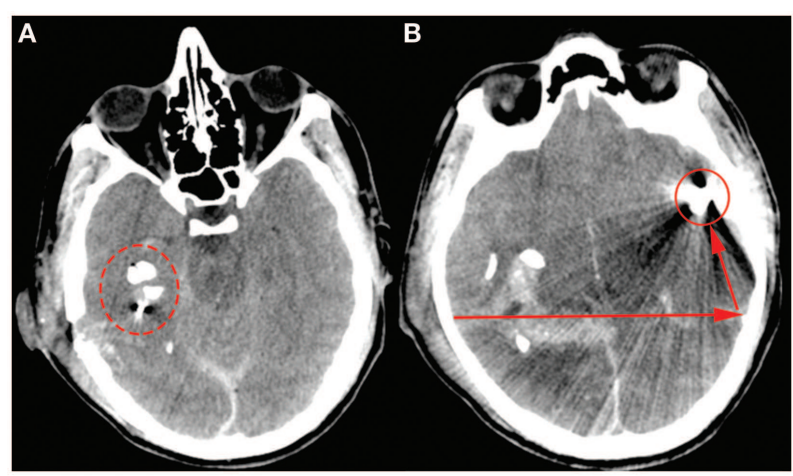

FIGURE 1 |The admission CT-scan, (A) highlights the bone fragments close to the entry point behind the right ear (ring). (B) lllustrates the bullet (ring) and its trajectory (arrows). neurosurgical clinic, the patient was still blind and had cognitive deficits yet had regained almost all motoric functions and could walk a shorter distance without support. Six months after trauma, the patient is still dependent on medical staff in order to move, as well as suffering from cortical blindness and cognitive shortfall.

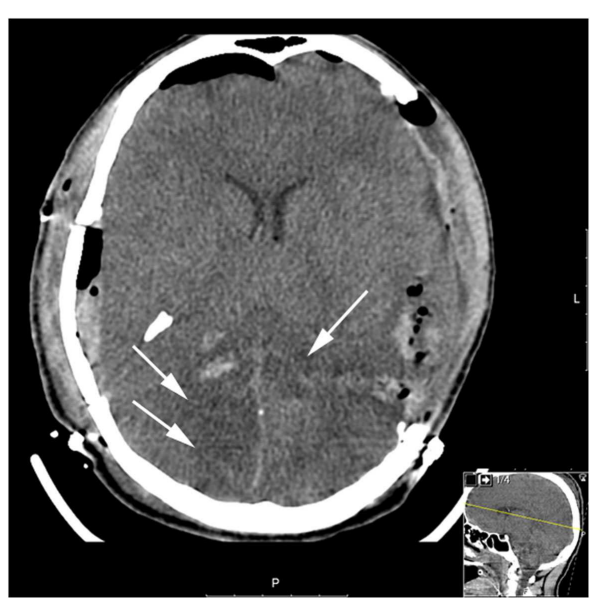

FIGURE 3 | Post-operative CT (after hemicraniectomy) day 2. The white arrows highlight the hypodense regions of brain parenchyma in the posterior circulation which is tissue at risk for subsequent ischemic injury.

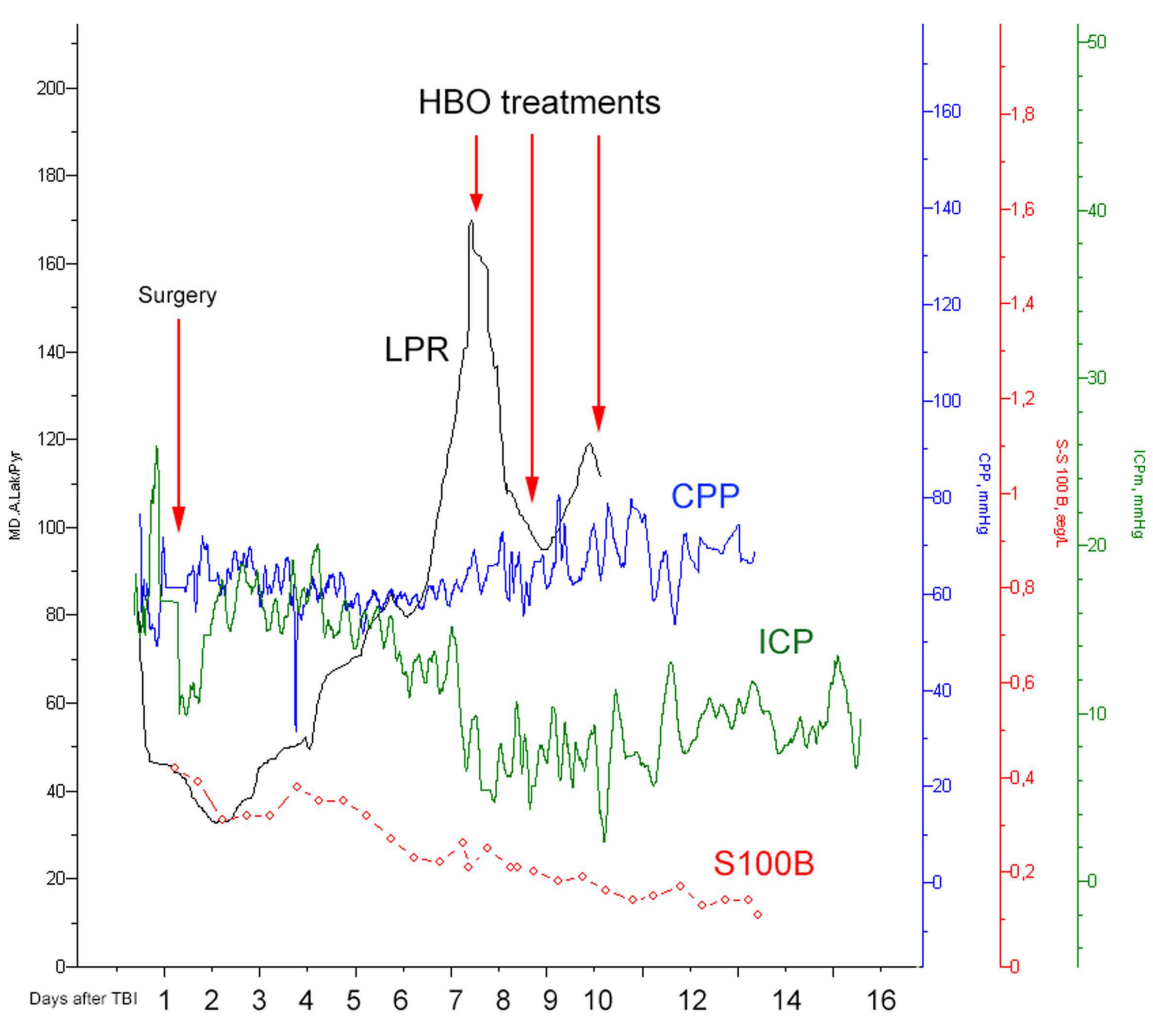

FIGURE 2 | Illustrating how the lactate:pyruvate ratio (LPR, black), the central perfusion pressure (CPP, blue), the intracranial pressure (ICP, green), and S100B (red) levels changed over time. The first red arrow indicates the time for hemicraniectomy ("surgery") while the other three highlight the timings for HBO treatment. 


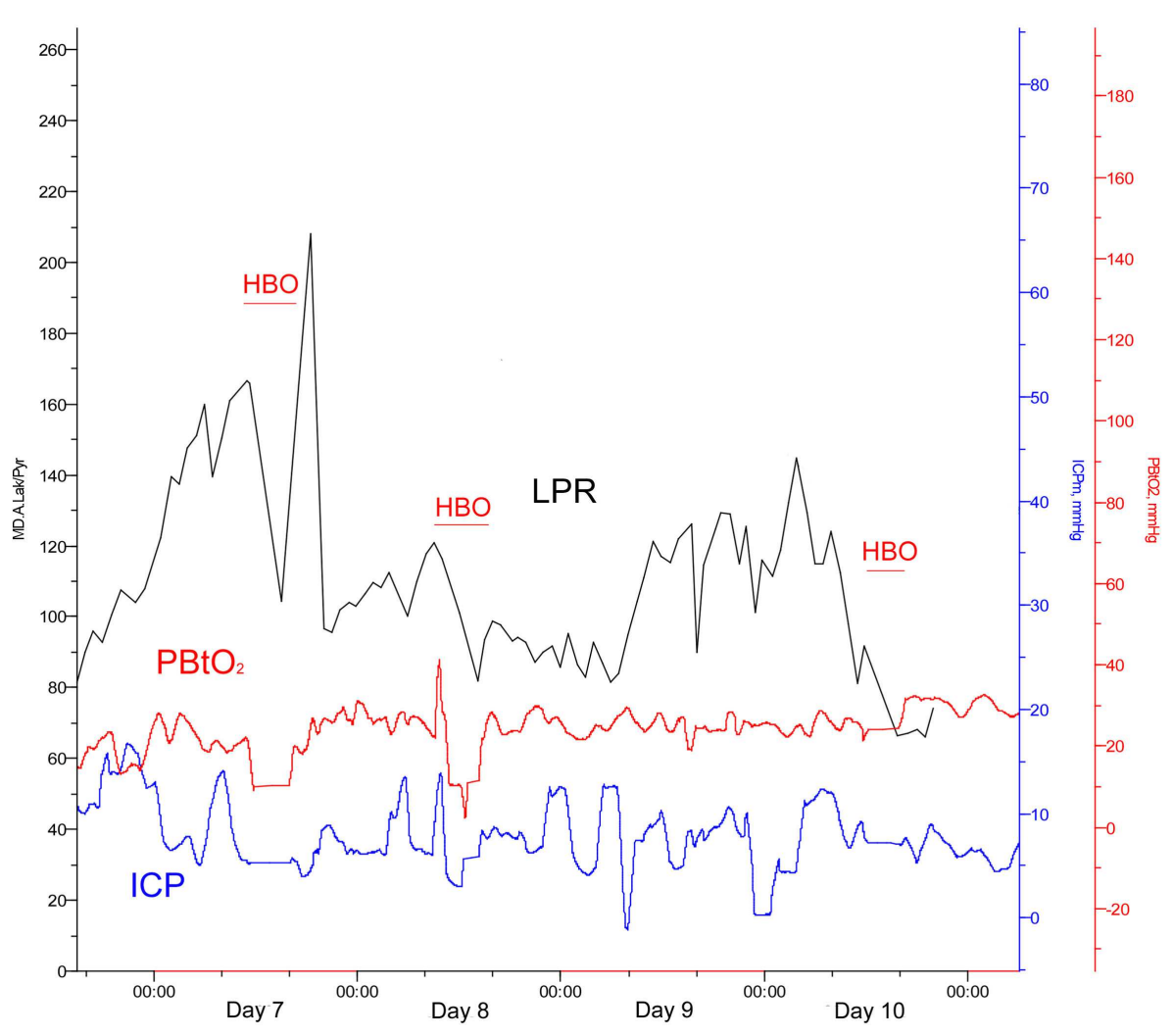

FIGURE 4 | Lactate:pyruvate ratio (LPR, black), intracranial pressure (ICP, blue), and the brain tissue oxygen pressure (PBtO2, red) changes during HBO treatment (red blocks).

\section{BACKGROUND}

It has been shown that patients with low GCS at admission, unresponsive pupils, bihemispheric transventricular injury, and subarachnoid hemorrhage usually have a poor outcome after cerebral gunshot wounds (1). This penetrating brain injury was treated as a severe traumatic brain injury (TBI) (GCS3-8). At our department, patients suffering from severe TBI are mechanically ventilated, and sedated with morphine, midazolam, and/or propofol. If mass lesions are present, they are evacuated as deemed appropriate. Mean arterial pressure (MAP) is measured intraarterially. Cerebral perfusion pressure (CPP) is calculated as MAPICP with the transducers placed at mid-lateral ventricular level. The patients are treated in $30^{\circ}$ sitting position. ICP is targeted at $<20 \mathrm{mmHg}$ (2) and CPP is targeted at $50-70 \mathrm{mmHg}$. Targets are achieved with intravenous infusions, vasopressors, osmotic therapy, and intermittent CSF drainage from ventricular catheters, ventilation, temperature control, and decompressive craniotomy, if needed. Patients are normoventilated $\left(\mathrm{pCO}_{2}\right.$ approx. $4.5 \mathrm{kPa}$ ). Blood glucose is targeted at $4-8 \mathrm{mmol} / \mathrm{L}$ and hemoglobin is targeted at $>90 \mathrm{~g} / \mathrm{L}$. Temperature is regulated at $37^{\circ} \mathrm{C}$ with paracetamol or external wrapping cooling systems. If ICP could not be regulated with the previously described techniques, sodium thiopental is infused, limited by burst suppression and monitored with continuous electroencephalography (EEG). Mild hypothermia $\left(35-36^{\circ} \mathrm{C}\right)$ is used for high refractory ICP.
Intracerebral microdialysis is performed routinely in patients suffering from severe traumatic brain injuries (3). Increasing LPR $>40$ indicates tissue ischemia or mitochondrial dysfunction. We routinely obtain serum samples of the biomarker S100B every $12 \mathrm{~h}$ and recognize secondary peaks as a negative prognostic indicator (4). Hyperbaric treatment seemed already in the mid1970 s to improve outcome in patients suffering from TBI presenting mid-brain symptoms (5), especially during 1.5-2.0 bar for 40 min (6). Recent studies by Rockswold and co-workers have shown beneficial effect by using hyperbaric treatment with significantly improved markers of oxidative metabolism, reduced intracranial hypertension, and improvement in markers of cerebral toxicity as well as a significant reduction in mortality and improved favorable outcome $(7,8)$.

In the present case report, we present a patient with relatively high GCS and normal pupil response at admission, despite a gunshot wound with a detrimental bihemispheric trajectory. The patient developed an increasing LPR, indicating local tissue damage due to ischemia or mitochondrial dysfunction, and was thus treated with 2.8 bar HBO. The intracranial conditions of the patient improved, and the patient survived despite unfavorable odds.

The current setting presents a higher pressure than reported by the Rockswold group (8). This regimen was chosen as a rescue measure of "last resort," facing the uncontrolled increase in LPR, because of presumed cerebral mitochondrial dysfunction. An 


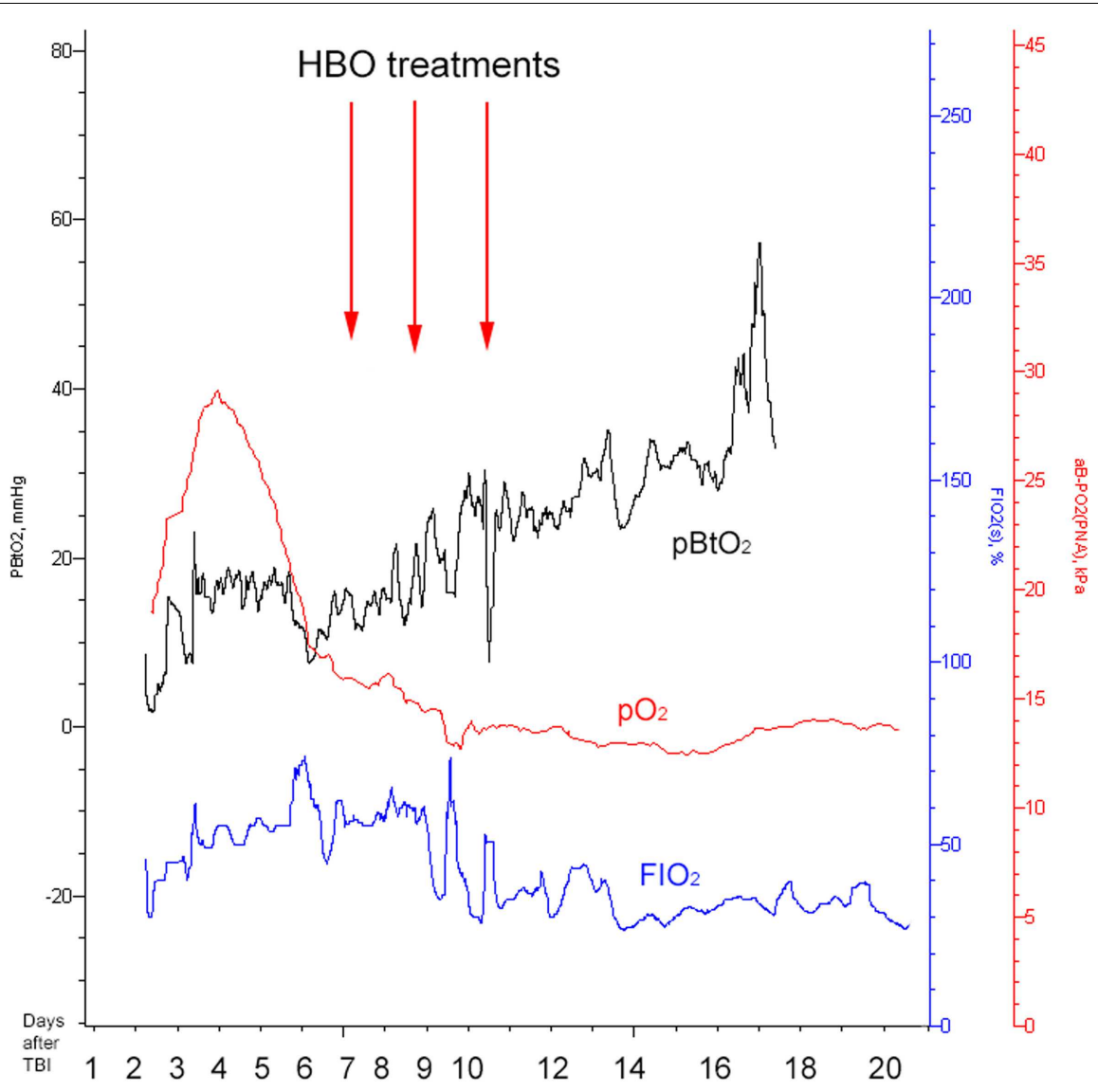

FIGURE 5 | Illustrating how the brain tissue oxygen pressure (PBtO2, black), the arterial blood gas oxygen pressure (pO2, red), and fraction of inspired oxygen (FIO2, blue) changed over time. The red arrows indicate timing for HBO treatments. All the measurements presented are performed at normobaric conditions (before and after $\mathrm{HBO}$ ).

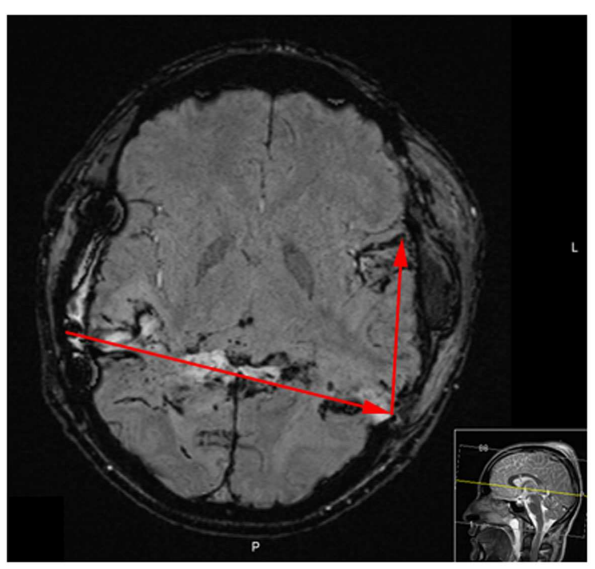

FIGURE 6 | MRI performed [susceptibility weighted imaging protocol (SWI)] on day 16 after injury reveals bilateral temporal damage and hemorrhage along the bullet, yet no signs of ischemic injury

increased $\mathrm{FIO}_{2}$ was tested, but failed to improve cerebral metabolic conditions (Figure 5). The patient's vital and metabolic parameters were thoroughly monitored during the $\mathrm{HBO}$ procedure, and the pressure would be lowered if any adverse effects had been detected. Despite the success achieved in this particular case, we do not recommend this regimen as a standard $\mathrm{HBO}$ procedure in severe TBI cases. More studies are necessary to determine the optimal dose regimen and to individualize the treatment.

The graphs presented are exported using the LabPilot ${ }^{\circledR}$ software (Microdialysis AB, Stockholm, Sweden).

\section{DISCUSSION}

Pathophysiology following TBI is complex, initiated by the primary damage to the brain parenchyma and a subsequent disintegration of the blood-brain barrier (BBB), leading to the development of cerebral edema. An early decrease in cerebral blood flow, hampering oxygen and substrate delivery to the cerebral tissue, leads to a subsequent development of ischemia, and eventually cellular death.

The ischemic environment present in the affected tissue, leads to an impaired mitochondrial function with ensuing anaerobic metabolism with increased intracerebral levels of lactate and increased LPR and an increased risk of development of secondary brain injuries $(9,10)$. However, a favorable environment in the border zone between dead and survivable tissue, may facilitate the recovery of the affected brain parenchyma, prevent negative effects 
of secondary insults, and by that improve patient outcome $(11,12)$. There are several animal models, and clinical TBI studies, elaborating the effects of normobaric hyperoxia (NBO) and $\mathrm{HBO}$ in TBI. Both $\mathrm{NBO}$ and $\mathrm{HBO}$ are capable of improving physiological variables, such as cerebral oxygenation (PBtO2) and metabolism (LPR) $(13,14)$. Experimental HBO treatments have been shown to improve mitochondrial function, increase ATP production, and reduce cell death in the hippocampus $(15,16)$. Recently, clinical data provided by professor Rockswold's group confirmed beneficial effects of $\mathrm{HBO}$ in human severe TBI, assessed from both surrogate endpoints, such as ICP, LPR, PBtO2, but also from a significant mortality reduction and an increase of favorable outcome (8). In our case, similar to the study by Rockswold et al., the intraparenchymal LPR decreased, the $\mathrm{PBtO} 2$ increased, and the ICP decreased after HBO (8). Also, the HBO treatment correlated to a decrease of serum S100B levels, where secondary increases often correlate to the development of ischemic injuries, as seen in a previous study by our group (4). Despite a secondary CT-scan revealing tissue at risk for further ischemic deterioration, as well as quickly deteriorating metabolic conditions, no further ischemic, or other, injury was visible on the MRI performed on day 16 .

It is important to note that the clinical course for each patient is highly individual. Even if the current HBO treatment improved conditions, its direct effect on outcome is not possible to evaluate from this particular case. The aim with multi-modal monitoring is to assess the effects of the individualized therapies provided for each patient. In this case, HBO treatment was followed by improved brain oxygenation and a better metabolic status (LPR), indicating a clinical usefulness.

The hyperbaric facility at Karolinska is equipped with a large multiplace chamber (HAUX, Germany). The working area of the chamber is $50 \mathrm{~m}^{2}$, and the HBO department is located adjacent to the ICU. The chamber is equipped with modern ICU-hardware, similar to the ordinary ICU, but approved for use during hyperbaric conditions. This makes it possible to provide $\mathrm{HBO}$ treatment to patients in circulatory and respiratory distress, such as toxic shock syndrome in case of necrotizing soft tissue infections. However, patients with TBI are not routinely treated in the hyperbaric chamber at our department. In special cases, HBO treatment could be considered. Future prospective studies should be launched to further validate the effect on outcome of HBO treatment in TBI.

\section{CONCLUSION}

In this case study, 2.8 bar HBO therapy improved the intracranial biochemical conditions of a patient suffering from a cerebral bihemispheric gunshot wound, indicating a potential benefit of HBO treatment. Further studies are warranted to better select which TBI patients that would best benefit from HBO treatment.

\section{REFERENCES}

1. Martins RS, Siqueira MG, Santos MT, Zanon-Collange N, Moraes OJ. Prognostic factors and treatment of penetrating gunshot wounds to the head. Surg Neurol (2003) 60:98-104. doi:10.1016/S0090-3019(03)00302-1

2. Bratton SL, Chestnut RM, Ghajar J, McConnell Hammond FF, Harris OA, Hartl R, et al. Guidelines for the management of severe traumatic brain injury. VIII. Intracranial pressure thresholds. J Neurotrauma (2007) 24:S55-8. doi:10.1089/neu.2007.9988
3. Bellander BM, Cantais E, Enblad P, Hutchinson P, Nordström CH, Robertson C, et al. Consensus meeting on microdialysis in neurointensive care. Intensive Care Med (2004) 30:2166-9. doi:10.1007/s00134-004-2461-8

4. Thelin EP, Nelson DW, Bellander BM. Secondary peaks of S100B in serum relate to subsequent radiological pathology in traumatic brain injury. Neurocrit Care (2014) 20:217-29. doi:10.1007/s12028-013-9916-0

5. Holbach KH, Wassmann H, Kolberg T. [Improved reversibility of the traumatic midbrain syndrome using hyperbaric oxygen]. Acta Neurochir (1974) 30:247-56. doi:10.1007/BF01405583

6. Holbach KH, Caroli A, Wassmann H. Cerebral energy metabolism in patients with brain lesions of normo- and hyperbaric oxygen pressures. J Neurol (1977) 217:17-30. doi:10.1007/BF00316313

7. Rockswold SB, Rockswold GL, Zaun DA, Zhang X, Cerra CE, Bergman TA, et al. A prospective, randomized clinical trial to compare the effect of hyperbaric to normobaric hyperoxia on cerebral metabolism, intracranial pressure, and oxygen toxicity in severe traumatic brain injury. J Neurosurg (2010) 112:1080-94. doi:10.3171/2009.7.JNS09363

8. Rockswold SB, Rockswold GL, Zaun DA, Liu J. A prospective, randomized Phase II clinical trial to evaluate the effect of combined hyperbaric and normobaric hyperoxia on cerebral metabolism, intracranial pressure, oxygen toxicity, and clinical outcome in severe traumatic brain injury. J Neurosurg (2013) 118:1317-28. doi:10.3171/2013.2.JNS121468

9. Chesnut RM, Marshall LF, Klauber MR, Blunt BA, Baldwin N, Eisenberg HM, et al. The role of secondary brain injury in determining outcome from severe head injury. J Trauma (1993) 34:216-22. doi:10.1097/00005373-19930200000006

10. Enriquez P, Bullock R. Molecular and cellular mechanisms in the pathophysiology of severe head injury. Curr Pharm Des (2004) 10:2131-43. doi:10.2174/ 1381612043384060

11. Bratton SL, Chestnut RM, Ghajar J, McConnell Hammond FF, Harris OA, Hartl R, et al. Guidelines for the management of severe traumatic brain injury. I. Blood pressure and oxygenation. J Neurotrauma (2007) 24:S7-13. doi:10.1089/neu.2007.9995

12. Werner C, Engelhard K. Pathophysiology of traumatic brain injury. Br J Anaesth (2007) 99:4-9. doi:10.1093/bja/aem131

13. Menzel M, Doppenberg EM, Zauner A, Soukup J, Reinert MM, Bullock R. Increased inspired oxygen concentration as a factor in improved brain tissue oxygenation and tissue lactate levels after severe human head injury. J Neurosurg (1999) 91:1-10. doi:10.3171/jns.1999.91.1.0001

14. Beynon C, Kiening KL, Orakcioglu B, Unterberg AW, Sakowitz OW. Brain tissue oxygen monitoring and hyperoxic treatment in patients with traumatic brain injury. J Neurotrauma (2012) 29:2109-23. doi:10.1089/neu.2012.2365

15. Daugherty WP, Levasseur JE, Sun D, Rockswold GL, Bullock MR. Effects of hyperbaric oxygen therapy on cerebral oxygenation and mitochondrial function following moderate lateral fluid-percussion injury in rats. J Neurosurg (2004) 101:499-504. doi:10.3171/jns.2004.101.3.0499

16. Zhou Z, Daugherty WP, Sun D, Levasseur JE, Altememi N, Hamm RJ, et al. Protection of mitochondrial function and improvement in cognitive recovery in rats treated with hyperbaric oxygen following lateral fluid-percussion injury. J Neurosurg (2007) 106:687-94. doi:10.3171/jns.2007.106.4.687

Conflict of Interest Statement: The authors declare that the research was conducted in the absence of any commercial or financial relationships that could be construed as a potential conflict of interest.

Received: 31 October 2014; accepted: 10 March 2015; published online: 23 March 2015. Citation: Thelin EP, Bellander B-M and Nekludov M (2015) Biochemical response to hyperbaric oxygen treatment of a transhemispheric penetrating cerebral gunshot injury. Front. Neurol. 6:62. doi: 10.3389/fneur.2015.00062

This article was submitted to Neurocritical and Neurohospitalist Care, a section of the journal Frontiers in Neurology.

Copyright (C) 2015 Thelin, Bellander and Nekludov. This is an open-access article distributed under the terms of the Creative Commons Attribution License (CC BY). The use, distribution or reproduction in other forums is permitted, provided the original author(s) or licensor are credited and that the original publication in this journal is cited, in accordance with accepted academic practice. No use, distribution or reproduction is permitted which does not comply with these terms. 